\title{
Tumor pH and Its Measurement
}

\author{
Xiaomeng Zhang ${ }^{1}$, Yuxiang Lin $^{2}$, and Robert J. Gillies ${ }^{3}$ \\ ${ }^{1}$ Biomedical Engineering Program, University of Arizona, Tucson, Arizona; ${ }^{2}$ College of Optical Sciences, University of Arizona, \\ Tucson, Arizona; and ${ }^{3}$ H. Lee Moffitt Cancer Center and Research Institute, Tampa, Florida
}

\begin{abstract}
Studies over the last few decades have demonstrated that the intracellular $\mathrm{pH}$ of solid tumors is maintained within a range of 7.0-7.2, whereas the extracellular $\mathrm{pH}$ is acidic. A low extracellular $\mathrm{pH}$ may be an important factor inducing more aggressive cancer phenotypes. Research into the causes and consequences of this acidic $\mathrm{pH}$ of tumors is highly dependent on accurate, precise, and reproducible measurements, and these have undergone great changes in the last decade. This review focuses on the most recent advances in the in vivo measurement of tumor $\mathrm{pH}$ by $\mathrm{pH}$-sensitive PET radiotracers, MR spectroscopy, $\mathrm{MRI}$, and optical imaging.
\end{abstract}

Key Words: pH; tumor; PET; MRS; MRI; optics

J Nucl Med 2010; 51:1167-1170

DOI: 10.2967/jnumed.109.068981

The physiologic microenvironment of solid tumors is normally characterized by poor perfusion and high metabolic rates. As a consequence, many regions within tumors are transiently or chronically hypoxic and acidic. Acidity is likely related to glucose consumption rates. High ${ }^{18} \mathrm{~F}-\mathrm{FDG}$ consumption has been consistently shown to be a poor prognostic indicator in human cancers. The $\mathrm{pH}$ of cells and tissues is the result of the balance between metabolic processes, proton transport, and buffering. Catabolism of sugars or fats results in a net production of acid, whether through fermentation or respiration (Fig. 1) (1). The pyruvate made by glycolysis or amino acids can either enter the tricarboxylic acid cycle through pyruvate dehydrogenase or be reduced to lactate. Carbonic acid arises by hydration of $\mathrm{CO}_{2}$ formed by oxidation in the tricarboxylic acid cycle, whereas lactic acid is the product of the glycolytic pathway in the absence of oxygen. Excess lactate is exported via monocarboxylate transporters 1,2 , and sometimes 4 , which can also carry $\mathrm{H}^{+}$. Protons themselves are exported via several transport systems such as $\mathrm{Na}^{+} / \mathrm{H}^{+}$ exchange, vacuolar $\mathrm{H}^{+}$adenosine triphosphatases, and $\mathrm{Na}^{+}$/ $\mathrm{HCO}_{3}{ }^{-}$exchanges. All 3 systems perform redundant functions and can substitute for one another in the presence of inhibitors. Oxidatively produced $\mathrm{CO}_{2}$ can exit the cell through

\footnotetext{
Received Dec. 15, 2009; revision accepted Mar. 4, 2010.

For correspondence or reprints contact: Robert J. Gillies; H. Lee Moffitt Cancer Center and Research Institute, 12902 Magnolia Dr., SRB-2, Tampa, FL 33612.

E-mail: Robert.Gillies@moffitt.org

COPYRIGHT (c) 2010 by the Society of Nuclear Medicine, Inc.
}

aquaporins, where it is hydrated by membrane-bound carbonic anhydrases, to yield bicarbonate and a hydrogen ion.

Maintenance of acid-base homeostasis is critical. However, this balance in solid tumors is vulnerable. In tumor cells, the increased glucose catabolism results in significant production of lactate and $\mathrm{H}^{+}$. Although tumor cells have increased acid production, they maintain a normal or alkaline intracellular $\mathrm{pH}(\mathrm{pHi})$ relative to normal cells. The major acid load is transported outside the cells, but the acid cannot be removed by the vasculature. The capacity of primary extracellular buffer is limited; as a result, extracellular space becomes acidic. Tumor cells seem well adapted to acidic microenvironments. In fact, in vitro studies have shown that tumor cell proliferation maximizes at an extracellular $\mathrm{pH}(\mathrm{pHe})$ of 6.8 instead of 7.3 in normal cells. The excess $\mathrm{H}^{+}$ions diffuse along concentration gradients from the tumor into adjacent normal tissue, resulting in a chronically acidic microenvironment for neighboring normal cells. Recent therapeutic approaches have been designed to target the tumor $\mathrm{pH}$ either through low-pH activation of pro-drugs, low-pH activation of drug release from micelles and nanoparticles, or drugs that raise the $\mathrm{pH}$ of acidic tumors. Thus, it will become increasingly important to be able to measure $\mathrm{pH}$ with accuracy, precision, and high spatiotemporal resolution in experimental preclinical systems and in human beings.

\section{PET}

PET has been used for measuring tissue $\mathrm{pH}$ since the 1970s. Techniques that have had moderate success use radiolabeled dimethadione, which distributes according to the $\mathrm{pH}$ gradient across semipermeable membranes via ion trapping. Although this technique represents the first noninvasive in vivo $\mathrm{pH}$ measurement, it is inaccurate and imprecise, since dimethadione distribution depends on the plasmalemmal $\mathrm{pH}$ gradient and the fractional volumes of intra- and extracellular space, both of which are unknown. Recently, an innovative technique was discovered to selectively target acidic tissues in vivo using $\mathrm{pH}$ (low) insertion peptide ( $\mathrm{pHLIP}$ ), a peptide that predominantly inserts across a lipid bilayer as a monomeric $\alpha$-helix at an acidic extracellular environment but not at a normal physiologic $\mathrm{pH}$. Vāvere et al. extended this method with PET to investigate the acidic environment in prostate tumors using ${ }^{64} \mathrm{Cu}$ conjugated to pHLIP $\left({ }^{64} \mathrm{Cu}\right.$-DOTA-pHLIP) (2). This was the first time a pHe-sensitive peptide-based PET agent was reported for delineating the pHe of tumors. Although the affinity of pHLIP to the cell membrane is low 


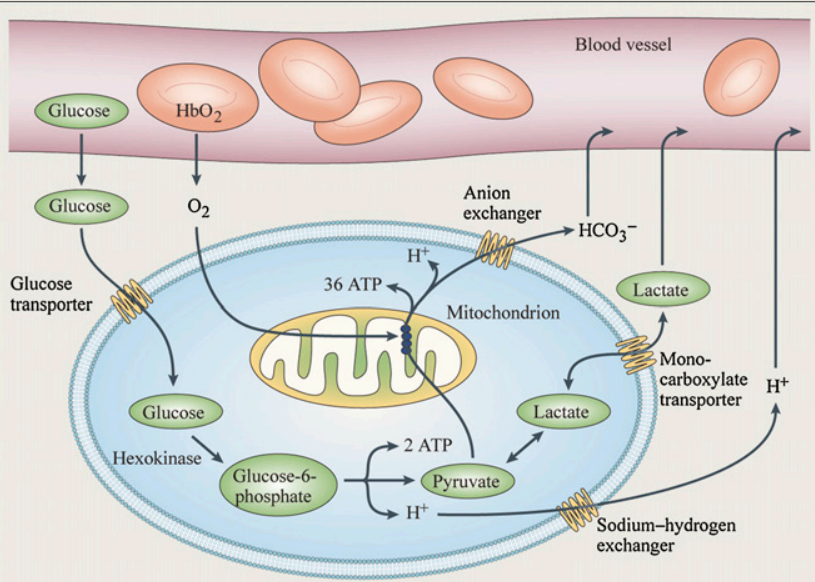

FIGURE 1. Glucose metabolism in mammalian cells. Afferent blood delivers glucose and oxygen (on hemoglobin) to tissues, where it reaches cells by diffusion. Glucose is taken up by specific transporters, where it is converted first to glucose-6-phosphate by hexokinase and then to pyruvate, generating 2 adenosine triphosphates per glucose. In presence of oxygen, pyruvate is oxidized to $\mathrm{HCO}_{3}{ }^{-}$, generating 36 additional adenosine triphosphates per glucose. In absence of oxygen, pyruvate is reduced to lactate, which is exported from cell. Both processes produce hydrogen ions $\left(\mathrm{H}^{+}\right)$, which acidify extracellular space. ATP = adenosine triphosphate; $\mathrm{HbO}_{2}=$ oxygenated hemoglobin. (Reprinted with permission of (1).)

at a normal physiologic $\mathrm{pH}$, an inaccurate $\mathrm{pHe}$ measurement may result.

\section{MR SPECTROSCOPY (MRS) AND MRI}

In vivo MRS and MRI have been used for more than 3 decades to monitor metabolic and physiologic processes. Both endogenous and exogenous nuclear MR-active compounds have been used to measure $\mathrm{pH}$ in vivo (3). MRS methods are generally based on a difference in chemical shifts between $\mathrm{pH}$ dependent and -independent resonances. Several isotopes have been evaluated to determine tissue $\mathrm{pH}$ with MRS. ${ }^{31} \mathrm{P}-\mathrm{MRS}$ provides a robust technique for simultaneously measuring $\mathrm{pHi}$ from the chemical shift of endogenous inorganic phosphate and $\mathrm{pHe}$ from the chemical shift of exogenous indicators, such as 3-aminopropyl phosphonate. Measurement was improved using ${ }^{1} \mathrm{H}$-MRS with the $\mathrm{pH}$-sensitive $\mathrm{H}_{2}$ resonance of 2-imidazole-1-yl-3-ethoxycarbonyl propionic acid. Although these studies showed that tumor pHe was heterogeneous, they are still limited in spatial and temporal resolution.

\section{Hyperpolarized ${ }^{13} \mathrm{C}$ Bicarbonate}

${ }^{13} \mathrm{C}$ is distinct from more commonly used isotopes in that it is only $1.1 \%$ naturally abundant. Hence, ${ }^{13} \mathrm{C}$ studies rely on using compounds with isotopic enrichment. Additionally, dynamic nuclear hyperpolarization can radically improve the sensitivity of in vivo ${ }^{13} \mathrm{C} \mathrm{MR}$. The technique is based on transferring the polarization of unpaired electrons to neighboring nuclei by microwave irradiation of the sample. Dynamic nuclear hyperpolarization has been shown to be capable of enhancing the MR signal of ${ }^{13} \mathrm{C}$ nuclear $\mathrm{MR}$ by more than a factor of 40,000. Brindle et al. have recently measured tumor $\mathrm{pH}$ using hyperpolarized ${ }^{13} \mathrm{C}$ bicarbonate (4). This measurement used the Henderson-Hasselbalch equation to estimate tissue $\mathrm{pH}$ from the voxelwise ratio of $\mathrm{H}^{13} \mathrm{CO}_{3}{ }^{-}$to ${ }^{13} \mathrm{CO}_{2}$ after injection of hyperpolarized $\mathrm{H}^{13} \mathrm{CO}_{3}{ }^{-}$. The results showed that the $\mathrm{pH}$ of a lymphoma xenograft was significantly lower $(6.7 \pm 0.1)$ than that of normal tissue $(7.1 \pm 0.1)$. Although a rapid pharmacokinetic distribution of bicarbonate measures a weighted average of $\mathrm{pHi}$ and $\mathrm{pHe}$, the results appear weighted to $\mathrm{pHe}$. A limitation of dynamic nuclear hyperpolarization is that the hyperpolarized nuclear spin signal decreases rapidly according to spin-lattice relaxation, T1. Therefore, measurements must be completed within 1-2 min after injection. Rapid relaxation also severely restricts the number of phase-encoding steps during short acquisitions, resulting in limited spatial resolution. However, fast imaging techniques such as compressed sensing reconstruction can be used to overcome these potential pitfalls (5).

\section{MR Relaxometry}

An alternative approach using MRI relies on perturbing the relaxivity of water via $\mathrm{pH}$-dependent relaxation agents. A smallmolecule approach to measuring $\mathrm{pH}$ has been developed by Sherry et al. (6) and Zhang et al (7), who have synthesized gadolinium-based agents whose relaxivity is $\mathrm{pH}$-dependent. In the case of the tetraphosphonate, gadolinium-DOTA-4AmP ${ }^{5-}$, the hydrogen-bonding network created by phosphonate sidearm protonation provides a catalytic pathway for hydrogen exchange. For quantification, this approach requires accurate knowledge of the agent concentration in each voxel. Raghunand solved that problem using sequential injection of 2 gadolinium agents, one of which was $\mathrm{pH}$-insensitive. This method has been applied to imaging $\mathrm{pH}$ in the kidneys and in rat brain gliomas (8). In the glioma model, the comparison of $\mathrm{pHe}$ and tumor perfusion time to maximal amplitude indicated that volumes with slower perfusion correlated with lower $\mathrm{pHe}$ values. Although this method worked well, there are drawbacks to the successive injection of 2 different agents, especially for potential clinical use. During the course of the injections, prolonged exposure to anesthesia may alter blood pressure, which can result in significant differences in the pharmacokinetics of successive injections. In addition, it is necessary to wait until most of the first agent has exited the tumor before the second is administered.

Recently, a relaxivity-based single-injection method has been developed, which consists of a mixture of dysprosiumDOTP $^{5-}$ with gadolinium-DOTA-4AmP ${ }^{5-}$ (9). Although the effects of the gadolinium contrast agent on $\mathrm{T} 1$ and $\mathrm{T} 2$ relaxation exhibited similar pHe dependence, the dysprosium contrast agent induced a strong outer-sphere effect on $\mathrm{T} 2 *$ that was pHe-independent, with negligible effect on T1. This method involved measurement of the concentration of dysprosium contrast agent through its effects on spin-spin relaxivity including magnetic field effects $\left(\Delta R^{2 *}\right)$ and subsequently extrapolating the concentration of gadolinium contrast agent from the known molar ratio of dysprosium and gadolinium contrast agent, thus enabling dynamic calculation of spatially localized unique $\mathrm{pHe}$ values. The protocol is outlined in Figure 2 and represents the first application of a contrast agent cocktail for the determination of spin lattice and susceptibility- 




FIGURE 2. Schematic overview of single-injection protocol. In vitro calibrations (upper panel) are used to define relationship between molar relaxivity of gadolinium-DOTA-4AmP5- and $\mathrm{pH}$. In vivo calibrations (lower panel) involve coinjection of $\mathrm{pH}$-independent gadoliniumdiethylenetriaminepentaacetic acid and dysprosium-DOTP. These data are used to define in vivo relationship between concentration of gadolinium contrast agent and echoplanar spectroscopic imaging-measured line width. In experiment, line width induced by coinjected concentration of dysprosium contrast agent is used to calculate per-pixel concentration of gadolinium-DOTA-4AmP5-, which is then combined with T1 values to calculate molar relaxivity and, hence, $\mathrm{pH}$ (9). CA = contrast agent; DOTP = 1,4,7,10-tetraazacyclododecane- $N, N^{\prime}$, $N^{\prime \prime}, N^{\prime \prime \prime}$-tetra(methylene phosphonic acid); DTPA = diethylenetriaminepentaacetic acid; EPSI = echoplanar spectroscopic imaging; $L W=$ line width; $R 1=$ spin-lattice relaxation rate $(R 1=1 / T 1) ; r_{1}=$ molar relaxivity.

induced transverse relaxation in this manner (9). The primary advantage of this protocol over previous studies is the rapidity of the pHe measurement, as high-resolution pHe maps can be obtained with approximately $0.20 \mathrm{mM}$ gadolinium-DOTA$4 \mathrm{AmP}^{5-}$ within $16 \mathrm{~min}$ after injection. Aime et al. created a peptide that changes between a helix and a random coil in a $\mathrm{pH}-$ dependent manner, which affects rotational correlation time and $\mathrm{T} 1$ relaxivity but has little effect on $\mathrm{T} 2$ relaxivity. This approach consists of measuring the ratio between $\mathrm{T} 1$ and $\mathrm{T} 2$ paramagnetic contribution to the water proton relaxation rate, providing an opportunity to measure $\mathrm{pH}$ in a concentration-independent fashion (10). In principle, the single-injection method is capable of yielding $\mathrm{pHe}$ maps within practical times in a clinical setting.

\section{Chemical Exchange Saturation Transfer (CEST)}

Compared with T1- and T2-based contrast agents, novel agents have been developed to generate contrast via CEST, particularly at high magnetic fields. The dynamic process of CEST can be described by a simple 2-pool chemical exchange model, wherein the magnetizations for a labile proton and bulk water are derived by 2 groups of Bloch equations coupled by chemical exchange. CEST is mediated by presaturating a resonance that is undergoing chemical exchange and measuring the effect on decreasing the abundant water signal. Besides general MRI parameters, exchangeable site concentrations, temperature, and endogenous tissue properties, CEST contrast also depends on $\mathrm{pH}$. In general, the exchange rate is slower at a low $\mathrm{pH}$ than at a high $\mathrm{pH}$ because of base catalysis of proton exchange. There are 3 main categories of CEST imaging: diamagnetic CEST, paramagnetic CEST, and amide proton transfer. Ward and Balaban first demonstrated the possibility of CEST imaging in which radiofrequency saturation was transferred from exchangeable solute protons to water (11). This resulted in further work characterizing the diamagnetic CEST $\mathrm{pH}$ measurements using high concentrations of 2 low-molec- ular-weight solutes as tracers in vivo. Aime et al. demonstrated that iopamidol can be used as a diamagnetic CEST agent for MRI to measure $\mathrm{pH}$ in a concentration-independent fashion, because iopamidol contains 2 chemical groups that have different pH-dependent CEST effects (12). Mori, van Zijl, et al. optimized methods to obtain accurate chemical exchange rates between water and - $\mathrm{NH}$ protons in vivo by avoiding magnetization transfer signal losses for solvent suppression. This achievement has led to characterization of amide proton transfer effects based on the magnetization exchange from labile endogenous amide protons to bulk water, which has been applied to humans (13). A limitation of CEST imaging is that it requires homogeneous magnetic fields, which are difficult to achieve in motile tissues. However, recent technologies allow voxel-byvoxel correction based on the fact that the CEST spectral width is independent of field inhomogeneity and that the CEST spectrum is only shifted.

Application of the CEST technique has been furthered by incorporating a paramagnetic center in the exchanging molecule, leading to increased chemical shift dispersions and, hence, increased sensitivities (14). Paramagnetic agents with enlarged chemical shifts (of $>50 \mathrm{ppm}$ ) provide the possibility of keeping the slow nuclear MR conditions at high exchange rates. Lanthanide-based paramagnetic complexes containing both highly shifted $\mathrm{pH}$-insensitive and $\mathrm{pH}$-sensitive exchangeable protons have been developed, wherein the CEST effects at 2 resonances can be used for a ratiometric determination of $\mathrm{pH}$. The drawbacks to CEST and paramagnetic CEST remain the high concentrations required $(>10 \mathrm{mM})$ and the need for strong MR irradiation pulses for presaturation, which are limited by power deposition. Pagel's group has developed a paramagnetic CEST agent, ytterbium-1,4,7,10-tetraazacyclododecane-1,4,7-tetraacetic acid, 10-oaminoanilide, with 2 $\mathrm{pH}$-responsive CEST effects that have different MR frequen- 


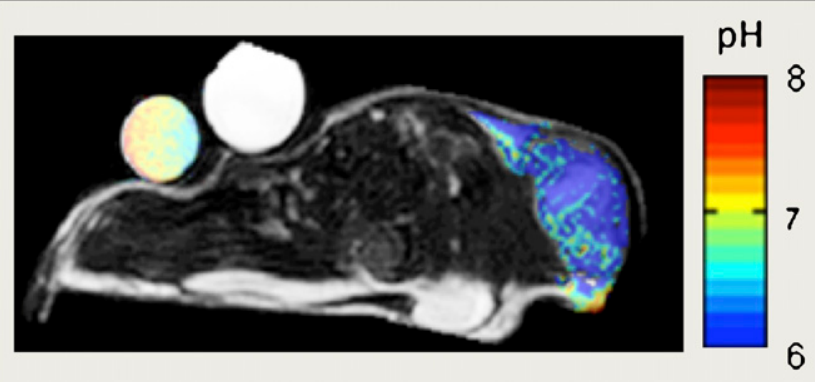

FIGURE 3. pHe map of mouse MCF-7 breast tumor model. $\mathrm{pH}$ was measured by paramagnetic CEST MRI with contrast agent ytterbium-1,4,7,10-tetraazacyclododecane-1,4,7-tetraacetic acid, 10-oaminoanilide. (Data courtesy of $\mathrm{M}$. Pagel (15).)

cies and different dependencies on $\mathrm{pH}$ (Fig. 3) (15). The ratio of the 2 paramagnetic CEST effects can measure the entire physiologic range of $\mathrm{pHe}$ from 6.1 to 8.0 with acceptable radiofrequency powers.

\section{OPTICS}

Optics are another powerful tool of noninvasive $\mathrm{pH}$ measurement in tumors and surrounding tissue. The varying fluorescent properties of the probes, which are associated with local $\mathrm{pH}$, can be measured optically and be converted to $\mathrm{pH}$ distribution accordingly. Currently, there are 2 main ways for measuring tumor $\mathrm{pH}$ : fluorescence ratio imaging microscopy and fluorescence lifetime imaging microscopy, which are accomplished by the measurement of emission spectra and lifetimes of fluorophores, respectively. In fluorescence ratiometric imaging, the emission spectra of some $\mathrm{pH}$ probes undergo a $\mathrm{pH}$-dependent wavelength shift, thus allowing the ratio of the fluorescence intensities from the dyes at 2 emission wavelengths to be used for accurate determinations of $\mathrm{pH}$. However, the association of $\mathrm{pH}$ indicators to intracellular proteins and cytosolic constituents was found to modify the ratio of intensities in 2 emission bands. Moreover, fluorescence ratio imaging microscopy is based on fluorescence amplitude methods and is thus susceptible to photobleaching and to variation in light scattering and absorption of the sample, which may also introduce bias in the results of $\mathrm{pH}$ measurement. Fluorescence lifetime imaging microscopy is an experimental technique in which the fluorescence decay is measured at each spatially resolvable location within a fluorescence image. The $\mathrm{pH}$ probes used in fluorescence lifetime imaging microscopy display a $\mathrm{pH}$-dependent shift in fluorescence lifetimes, which can be converted to a $\mathrm{pH}$ map of the tumor accordingly with construction of proper calibration curves. Fluorescence lifetime techniques are generally classified into 2 types: time domain and frequency domain. Compared with frequency domain methods, time domain measurements have a higher signal-to-noise ratio and are widely used for $\mathrm{pH}$ imaging (16). Although lifetime-based $\mathrm{pH}$ imaging has often been found to be more convenient than conventional ratiometric methods because of a simpler $\mathrm{pH}$ calibration, this method is not necessarily a straightforward approach to measuring $\mathrm{pH}$ in the absence of correction for the effect of probe binding.

\section{SUMMARY}

Techniques for measuring the $\mathrm{pH}$ of organelles, cytosol, and extracellular fluid in vivo have been consistently improving and converging. Optical techniques based on expression of $\mathrm{pH}-$ sensitive fluorescent dye are revolutionizing $\mathrm{pH}$ measurements in vitro. With improved detection platforms, optical methods hold promise to be applied in vivo. MR techniques have been developed to measure $\mathrm{pHi}$ with endogenous indicators, albeit with low sensitivity. Methods based on exogenous compounds have clearly shown that high resolution and highly sensitivity measurements of $\mathrm{pHe}$ (and possibly $\mathrm{pHi}$ ) are tractable in vivo. Hyperpolarized ${ }^{13} \mathrm{C}$-labeled bicarbonate techniques may potentially be used in the clinic, and new generations of $\mathrm{pH}$ sensitive PET tracers are under improvement. All these developments are a testament to the emerging view that tissue $\mathrm{pH}$ is a biomedically important parameter of tumor metabolism.

\section{REFERENCES}

1. Gatenby RA, Gillies RJ. Why do cancers have high aerobic glycolysis? Nat Rev Cancer. 2004;4:891-899.

2. Vāvere AL, Biddlecombe GB, Spees WM, et al. A novel technology for the imaging of acidic prostate tumors by positron emission tomography. Cancer Res. 2009;69:4510-4516.

3. Gillies RJ, Morse DL. In vivo magnetic resonance spectroscopy in cancer. Annu Rev Biomed Eng. 2005;7:287-326.

4. Gallagher FA, Kettunen MI, Day SE, et al. Magnetic resonance imaging of $\mathrm{pH}$ in vivo using hyperpolarized ${ }^{13} \mathrm{C}$-labelled bicarbonate. Nature. 2008;453:940943.

5. Hu S, Lustig M, Chen AP, et al. Compressed sensing for resolution enhancement of hyperpolarized ${ }^{13} \mathrm{C}$ flyback 3D-MRSI. J Magn Reson. 2008;192:258-264.

6. Aime S, Botta M, Crich SG, Giovenzana G, Palmisano G, Sisti M. A macromolecular $\mathrm{Gd}(\mathrm{III})$ complex as $\mathrm{pH}$-responsive relaxometric probe for MRI applications. Chem Commun (Camb). 1999;16:1577-1578.

7. Zhang S, Wu K, Sherry AD. A novel pH-sensitive MRI contrast agent. Angew Chem Int Ed Engl. 1999;38:3192-3194.

8. Garcia-Martin ML, Martinez GV, Raghunand N, Sherry AD, Zhang SR, Gillies RJ. High resolution $\mathrm{pH}(\mathrm{e})$ imaging of rat glioma using $\mathrm{pH}$-dependent relaxivity. Magn Reson Med. 2006;55:309-315.

9. Zhang X, Martinez GV, Garcia-Martin ML, Woods M, Sherry D, Gillies RJ. High spatio-temporal resolution $\mathrm{pHe}$ mapping of a rat glioma derived from $\mathrm{pH}$ dependent spin-lattice relaxivity. Presented at: ISMRM; May 3-9, 2008; Toronto, Ontario, Canada.

10. Aime S, Fedeli F, Sanino A, Terreno EA. R-2/R-1 ratiometric procedure for a concentration-independent, pH-responsive, Gd(III)-based MRI agent. J Am Chem Soc. 2006;128:11326-11327.

11. Ward KM, Balaban RS. Determination of $\mathrm{pH}$ using water protons and chemical exchange dependent saturation transfer (CEST). Magn Reson Med. 2000;44: 799-802.

12. Aime S, Calabi L, Biondi L, et al. Iopamidol: exploring the potential use of a well-established x-ray contrast agent for MRI. Magn Reson Med. 2005;53:830834.

13. Zhou J, Blakeley JO, Hua J, et al. Practical data acquisition method for human brain tumor amide proton transfer (APT) imaging. Magn Reson Med. 2008; 60:842-849.

14. Aime S, Barge A, Castelli DD, et al. Paramagnetic lanthanide(III) complexes as pH-sensitive chemical exchange saturation transfer (CEST) contrast agents for MRI applications. Magn Reson Med. 2002;47:639-648.

15. Liu G, Li Y, Pagel M. A single PARACEST MRI contrast agent for accurate in vivo $\mathrm{pH}$ measurements. Presented at the annual meeting of the International Society for Magnetic Resonance in Medicine; Berlin, Germany; May 2007.

16. Hassan M, Riley J, Chernomordik V, et al. Fluorescence lifetime imaging system for in vivo studies. Mol Imaging. 2007;6:229-236. 